\title{
ANÁLISIS SOCIOLINGÜÍSTICO DE LA ENTONACIÓN EN ENUNCIADOS DECLARATIVOS DEL ESPAÑOL DE CUBA Y ESPAÑA: PRIMER ACERCAMIENTO AL CORPUS PRESEEA
}

\author{
Adriana Pedrosa Ramírez $z^{38}$ \\ Madeleyne Bermúdez Sánchez ${ }^{39}$ \\ Raquel María García Riverón ${ }^{40}$
}

RESUMEN: En este artículo se presentan la descripción y el análisis de la entonación en enunciados declarativos atendiendo a las variables sociolingüísticas sexo y edad. La muestra analizada corresponde al español hablado en Cuba y al español de España recogidos en el corpus PRESEEA. Para la representación prosódica se emplea el sistema de transcripción sp-tobi en el marco teórico-metodológico del modelo métrico autosegmental. En términos generales, se encuentran dos configuraciones prosódicas del tonema en ambas variantes del español: $\mathrm{L}+\mathrm{H}^{*} \mathrm{~L} \%$ en enunciados largos paroxítonos y $\mathrm{L}^{*} \mathrm{~L} \%$ en enunciados cortos oxítonos.

Palabras clave: dialecto; prosodia; enunciados declarativos; tonema; modelo métrico autosegmental.

ABSTRACT: Neste artigo apresentamos a descrição e a análise da entonação em enunciados declarativos baseadas nas variáveis sociolinguísticas sexo e idade. A amostra analisada corresponde ao espanhol falado em Cuba e ao espanhol da Espanha, coletados no corpus PRESEEA. Para a representação prosódica, empregamos o sistema de transcrição sp-tobi no referencial teórico-metodológico do modelo métrico auto-segmentar. Em termos gerais, encontramos duas

38 Máster en Estudios Lingüístico-Editoriales Hispánicos. Profesora auxiliar del Departamento de Lingüística y Literatura de la Universidad Central'Marta Abreu' de Las Villas-Cuba.

${ }^{39}$ Doctora en Ciencias Lingüísticas. Profesora auxiliar del Departamento de Lingüística y Literatura de la Universidad Central'Marta Abreu' de Las Villas-Cuba.

${ }^{40}$ Doctora en Filología Hispánica. Profesora Titular de la Universidad de La Habana-Cuba. 
configurações prosódicas do tonema em ambas as variantes do espanhol: $\mathrm{L}+\mathrm{H}^{*} \mathrm{~L} \%$ em enunciados longos paroxítonos e $\mathrm{L}{ }^{*} \mathrm{~L} \%$ em enunciados curtos oxítonos..

Keywords: dialeto; prosódia; enunciados declarativos; tonema; modelo métrico autosegmental.

La variación dialectal y sociolingüística de la entonación española. Principales estudios y proyectos

Desde la segunda mitad del siglo XX se abre un espectro de corrientes lingüísticas que establecen vínculos con diferentes ramas o áreas del saber. Esta postura (inter) transdisciplinar supone el estudio del lenguaje en sus vínculos con el contexto o situación comunicativa, lo cual supone la inclusión de variables o aspectos extralingüísticos que influyen decisivamente en la producción e interpretación de un enunciado determinado. En todos los niveles de la lengua opera esta relación y, por tanto, también la entonación es reflejo de estos vínculos.

Así lo demuestran los estudios sociolingüísticos y dialectales de la entonación que exponen las relaciones en sus análisis entre la lengua y las variables sociales y también con una perspectiva geográfica, extensional. Entre los autores que han desarrollado esta perspectiva de análisis cabe destacar en el español peninsular a Quilis (1989), Moreno (1998, 1999), Sosa (1999), Revert Sanz (2001), Dorta (2007 a y b, 2013, 2018), y en el ámbito hispanoamericano a García Riverón (1985, 1996-1998), Martín Butragueño (2004, 2011), Velásquez Upegui (2013) y Muñoz Alvarado (2013).

Ya desde finales de la década de 1980 Antonio Quilis (1989) estableció los tres niveles en que puede actuar la entonación, entre ellos, el nivel sociolingüístico, en el que la entonación comunica dos informaciones: una relacionada con las características personales (edad, sexo, carácter) y otra que comunica el origen geográfico, el medio social y el nivel cultural al que pertenece el individuo. Por su parte, para Moreno Fernández (1998):

Algunos aspectos del uso social de la lengua en los que la entonación se revela como cardinal, al margen de las funciones puramente lingüísticas -intralingüísticas, si se 
Análisis sociolingüístico de la entonación en enunciados declarativos del español de Cuba y España: primer acercamiento al corpus preseea| 107

quiere- podrían ser la expresión de intenciones comunicativas, de inferencias, de marcas de discurso o conversacionales, la expresión de marcas de grupos geolectales y sociales, de marcas de estilo y de registro, así como la producción y comprensión de lenguas extranjeras (p. 1).

Entre los trabajos más notorios relacionados con la entonación dialectal se presenta el de Sosa (1999), para quien "uno de los factores más característicos y resaltantes que permite inmediatamente a un hablante identificar el origen geográfico de su interlocutor es precisamente la entonación” (p. 177). En esta investigación, además de presentar presupuestos generales sobre dialectología y sociolingüística y sus vínculos con el estudio de la entonación, se describe la prosodia de varios dialectos, entre los que se encuentran Madrid, Barcelona, Pamplona, Sevilla y en el español hispanoamericano los dialectos de las capitales de Argentina, Colombia, México, Puerto Rico, Venezuela, Cuba y Perú.

Considerando estas premisas donde se vincula el estudio de la entonación con la expresión de marcas geolectales o sociales, muchos de los estudios de la entonación española en las últimas décadas están enfocados hacia la confección del Atlas Multimedia de la Prosodia del Espacio Románico (AMPER en España) que como macroproyecto internacional para todas las variedades de lengua románica fue presentado por Manuel Contini en 1991. Este proyecto tiene como finalidad estudiar los rasgos prosódicos: análisis de la estructura entonativa y los parámetros temporales y de intensidad de las distintas variedades geolingüísticas en frases enunciativas e interrogativas de todas las lenguas románicas. Aunque inicialmente el proyecto se circunscribió a Europa, luego se sumaron un grupo en Brasil para el análisis de las variedades americanas del portugués, otros dos grupos en Chile y otro en Cuba que asumieron el estudio de la prosodia en el territorio americano.

En el ámbito latinoamericano sobresale también el proyecto COEM (Corpus oral del español de México), coordinado por Pedro Martín Butragueño, Leonor Orozco y José Esteban Hernández. En este proyecto se estudian las hablas mexicanas y se incluye el estudio de la prosodia atendiendo a las características geográficas y sociolingüísticas de las diferentes comunidades de México. 
En Cuba, por su parte, como afirma García Riverón (1985) “son escasos los trabajos dedicados al estudio de la variante sociolingüística de la entonación, aunque es frecuente encontrar rasgos suprasegmentales marcados socialmente" y continúa insistiendo: "Fenómenos similares deben ser estudiados, siguiendo los métodos de análisis y cómputo de la sociolingüística actual” (p. 263).

En los estudios sobre determinadas variantes geolectales del español de Cuba, Curbeira Palomo (2002), para la entonación de Guantánamo y Muñoz Alvarado (2013), para la entonación de Santiago de Cuba, incluyen variables sociolingüísticas. También, considerando las características geográficas, se estudian y comparan la entonación de Cuba y España. Por ejemplo, Rodríguez César (2005), inspirada por la ausencia de estudios comparativos entre el sistema entonativo de Cuba y el de otras regiones de habla hispana, realizó un análisis de la interrogación absoluta en Cuba y España con un enfoque acústico y pragmático. Con esta misma visión, el trabajo de Pedrosa Ramírez (2007) contiene un estudio sobre la entonación emotiva del español peninsular en comparación con el sistema entonativo cubano, a partir de una muestra de los medios de comunicación televisivos.

Asimismo, desde los primeros esbozos para la conformación del Atlas lingüístico de Cuba (años 80) se han considerado entre las variables: sexo, nivel de instrucción, edad y ubicación geográfica. Esos planteamientos iniciales vieron la luz casi tres décadas después y sobre la base del sistema entonativo descrito por García Riverón (1996b y 1998) se confeccionó una encuesta aplicada en puntos poblados urbanos de 11 provincias del país. Dentro de los resultados fundamentales que arrojaron las investigaciones para la conformación del Atlas lingüistico de Cuba (2013) en su apartado dedicado a la entonación, se encuentran:

Los entonemas que presentan un mayor nivel de codificación en nuestra variante de lengua, por constatarse su funcionamiento en el 100 $\%$ de las encuestas aplicadas son el E-1, E-2, E-3, E-5 y E-7, que expresan valores modales de enunciación e interrogación neutral, no conclusión y llamada, respectivamente.

El valor comunicativo de la enunciación con advertencia se realiza en un porciento elevado con la VE-1a, definida en el sistema. Sin embargo, el E-1 también adopta esta función en contextos similares, En estos casos, la advertencia no está dada solo por la entonación, sino por las condiciones pragmáticas de uso. 
Análisis sociolingüístico de la entonación en enunciados declarativos del español de Cuba y España: primer acercamiento al corpus preseea| 109

No se registró la VE-1a en las provincias orientales de Holguín y Bayamo y en Sancti Spíritus la mayoría de los informantes alterna ambos entonemas.

La prosodia en enunciados declarativos a partir del estudio de las variantes peninsulares e hispanoamericanas

En el mencionado trabajo de Sosa (1999), donde se comparan varios dialectos de la Península y de Hispanoamérica, sobresalen resultados que afirman que los enunciados declarativos absolutos presentan regularmente el tonema $\mathrm{L}^{*} \mathrm{~L} \%$ en todos los dialectos analizados; sin embargo, advierte también que en la ciudad de Bogotá, México, Caracas y Barcelona se presenta el tono $\mathrm{H}^{*} \mathrm{~L} \%$.

Uno de los trabajos que se realizan atendiendo a los presupuestos teórico-metodológicos del proyecto AMPER es el de Calleja Aspiazu (2004). Este análisis de los acentos tonales en el castellano de Vitoria se compara, además, con otros dialectos del castellano que se han analizado anteriormente: el castellano de Madrid y el de Lekeitio. Calculando las medias para el comienzo de la subida y para el pico, se llevó a cabo un análisis estadístico para encontrar correlaciones entre los valles y los picos, así como la posición de la sílaba tónica en la frase y el número de sílabas que precedían o seguían a la sílaba tónica en un corpus total de 45 oraciones. Calleja concluye en su artículo que la característica principal del castellano de Vitoria es que "en las sílabas tónicas hay una subida tonal y que esta subida acaba en la sílaba postónica. Pocas veces acaba la subida dentro de la sílaba tónica (sólo en final de oración)" (2004, p.47).

También dentro del proyecto AMPER, López Bobo, Cuevas Alonso, Díaz Gómez y Muñiz Cachón (2005) realizan la descripción y comparación de la estructura prosódica del asturiano hablado en la zona central (habla de Mieres y de Oviedo), a partir de una muestra obtenida por medio de dos informantes. Se describe en este artículo la prosodia de ambas variedades de asturiano, con lo que se muestran las diferencias y semejanzas que presentan, atendiendo no solo al acento léxico y acento entonativo, sino también considerando los valores de intensidad y duración para el estudio de la prosodia en la muestra seleccionada.

En relación con las diferencias entonativas entre las modalidades asertiva e interrogativa en el habla de Mieres y de Oviedo urbano, López Boboet al (2005) señalan la existencia de un patrón final de cadencia no 
sólo en los enunciados asertivos, sino también en los interrogativos. En relación a esto concluye: "En cuanto a la evolución global de la curva de entonación en asertivas e interrogativas se observa que es bastante similar en ambas modalidades, aunque las interrogativas presentan una frecuencia fundamental más alta que las asertivas. Esta diferencia es mucho más acusada en el habla de Mieres que en la de Oviedo, donde los valores máximos y mínimos son menos extremos" (p.196).

Este estudio concluye que los enunciados aseverativos presentan dos configuraciones $\mathrm{L}^{+}\left(_{j}\right) \mathrm{H}^{*} \mathrm{~L}^{*}$ en oriente y occidente urbano y en la zona rural muestran $\mathrm{H}+\mathrm{L}^{*} \mathrm{~L} \%$; además, el tono $\mathrm{L}+>\mathrm{H}^{*}$ es el pretonema más frecuente.

Por su parte, en el trabajo de Ramírez Verdugo (2005) se establece una taxonomía fonética y fonológica de los rasgos prosódicos característicos del habla de Madrid. Utilizando la metodología que establece el AMPER esta autora estudia la prosodia madrileña en oraciones enunciativas e interrogativas e incluye los acentos: agudos, llanos y esdrújulos. La investigación concluye que tanto en las oraciones enunciativas como en interrogativas absolutas se detectó mayor variedad en la realización de los acentos tonales de la que se había reconocido en estudios anteriores como en los de Quilis (1993, 1997), Sosa (1999), siendo la estructura tonal más común $\mathrm{L}^{*}+\mathrm{H}$ y $\mathrm{H}^{*}+\mathrm{L}$.

También dentro del AMPER, González Olivera, Simón Casas, Castañer Martín (2007) realizan un análisis comparativo de la prosodia de Zaragoza y Jaca. Este estudio concluye que la línea melódica en las oraciones enunciativas es similar en ambas localidades; sin embargo, hay diferencias en el tonema, con un descenso mucho más marcado en Zaragoza que en Jaca, población en la que destaca, además, la pequeña elevación que se produce en el valor final de frase.

Asimismo, Dorta (2007), describe la entonación de Canarias y establece relaciones con trabajos anteriores y con las variedades de lengua caribeñas. En la descripción se advierte que lo normal es la posrealización del primer pico y, por tanto, la no coincidencia de este y el acento léxico, hecho que había descrito ya Sosa (1999) para el español hispanoamericano. Además, en el corpus de análisis, se concluye que las declarativas de Gran Canaria, a diferencia de lo que había señalado Quilis (1989), como las de las demás islas como Tenerife y La Gomera, no se caracterizan por un movimiento circunflejo en el núcleo final. 
Análisis sociolingüístico de la entonación en enunciados declarativos del español de Cuba y España: primer acercamiento al corpus preseea| 111

En un estudio posterior, Martín y Dorta (2018) ofrecen las características de las declarativas e interrogativas en el español de Cuba a partir de las muestras de informantes en tres puntos de la geografía cubana: La Habana, Santa Clara y Santiago de Cuba. El estudio concluye que las oraciones declarativas cubanas comienzan generalmente con un tono $/ \% \mathrm{M} /$ en mujeres y hombres; por su parte, en el acento inicial las mujeres desplazan el pico inicial $\left[\mathrm{L}^{+}>\mathrm{H}^{*}\right]$ y los hombres lo sincronizan con la acentuada $\left[\mathrm{L}+\mathrm{H}^{*}\right]$ y en cuanto al patrón final de las declarativas se reconoce el $/ \mathrm{L}^{*} \mathrm{~L} \%$ coincidiendo con el patrón general del español.

Como se ha explicado anteriormente, en equilibrio con el panorama de estudios de carácter geolectal, donde se muestran los intereses por describir la entonación en todo el ámbito hispánico, afloran los estudios (aunque no tan numerosos) en Cuba que consideran la entonación en sus vínculos con características geográficas y sociales.

De este modo, se presenta la investigación de Curbeira Palomo (2002) basada en un análisis tonal que parte de la representación de la muestra obtenida en las grabaciones en el pentagrama musical ${ }^{41}$, teniendo en cuenta que la entonación y el canto se cimientan en los mismos principios esenciales, según como lo considera Tomás Navarro Tomás en sus estudios. ${ }^{42}$ Aplicando el método de análisis comunicativo fueron comparadas las unidades de entonación encontradas en el habla popular de la ciudad de Guantánamo con el sistema entonativo de la Ciudad de La Habana, descrito por García Riverón (1996a y b, 1998). Se hallaron también unidades entonativas que difieren de dicho sistema y que se reconocieron en la investigación como patrones entonativos (P-1G, P-2G, P-3G, P-4G). Considerando, además, las variables sociales de edad y sexo, se establecieron diferencias estadísticas significativas entre las diferentes generaciones que se sometieron a estudio, y también entre hombres y mujeres atendiendo a la frecuencia de realización de las unidades entonativas.

${ }^{41}$ Esta representación en el pentagrama fue realizada con la ayuda de músicos que de manera individual llevaron al piano las unidades segmentadas por la autora.

${ }^{42}$ Para conocer más sobre la relación entre canto y entonación pueden consultarse los criterios que María del Carmen Curbeira reseña en esta tesis $(2002$, p.17). 
Por su parte, siguiendo el enfoque fonológico estructural de la entonación y análisis melódico del habla propuesto por Cantero Serena y su grupo de investigación, la tesis doctoral de Muñoz Alvarado (2013) caracteriza la entonación coloquial de los universitarios santiagueros. En una muestra espontánea integrada por 72 informantes nativos de la ciudad de Santiago de Cuba, divididos en tres grupos etarios y con igual cantidad de mujeres y hombres por grupo, se procedió a la caracterización fonética de 500 contornos sometidos a análisis acústico y estandarización y se establecieron los patrones melódicos presentes en la norma entonativa estudiada, a saber: el entonema neutro: / interrogativo, - enfático, suspendido/, entonema simple interrogativo: /+ interrogativo, - enfático, enfático/, entonema simplemente suspendido: / interrogativo, -enfático, + suspendido/, entonema simplemente enfático: / interrogativo, + enfático, suspendido/.

Los otros cuatro entonemas determinados son denominados entonemas terciarios pues conjugan rasgos fonológicos de los ya descritos, de modo que su estructura melódica resulta la imbricación de rasgos fonéticos antes señalados. Aquí se distinguen: el entonema enfático suspendido, entonema interrogativo suspendido, entonema interrogativo enfático y el interrogativo enfático suspendido.

En la caracterización del entonema neutro: / interrogativo, - enfático, suspendido/, se establecen tres patrones melódicos, caracterizados por un final descendente o plano. Según se refiere, este entonema aparece para formular frases o enunciados declarativos acabados y que no implican expresividad o emoción.

El presente trabajo se orienta a la descripción de las características de los enunciados declarativos en el español hablado en Cuba y España, considerando para su estudio el Corpus del Proyecto para el estudio sociolingüístico del español de España y de América (PRESEEA). Para ello se describen las características generales de la entonación en las declarativas de ambas variantes de lengua a partir de la configuración del tonema y las particularidades dialectales asociadas con cada configuración.

\section{Corpus e informantes}

Se trabajó con el corpus del Proyecto para el estudio sociolingüístico del español de España y de América (PRESEEA). Entre 
las características de este tipo de corpus podemos señalar que es un corpus semiespontáneo, elaborado a partir de entrevistas y organizado teniendo en cuenta variables sociolingüísticas. Recoge una muestra por afijación uniforme, con lo que se reconoce por cada comunidad el mismo número de informantes según el sexo, el grupo etario a que pertenecen y el nivel de instrucción.

Se seleccionaron para este trabajo una muestra conformada por 6 informantes, tres de Cuba y 3 de España; dos son hombres y cuatro mujeres. Se analizaron 10 minutos de grabación por cada informante, todos con nivel de instrucción bajo.

Luego de un análisis auditivo riguroso de la muestra fue conformado un corpus a partir de la segmentación de 35 unidades declarativas que fueron procesadas acústicamente. Una de las dificultades para el estudio es la variedad en la extensión de las unidades objeto de análisis, pues cuanto más extensa es la unidad, más variedad y complejidad se enfrenta en el estudio de los datos. Tratando de homogeneizar el corpus de trabajo fueron divididos los enunciados en cortos (unidades de hasta 6 sílabas) y largos (unidades con más de 6 sílabas), oxítonos y paroxítonos, pudiendo establecer regularidades en enunciados cortos oxítonos y en enunciados largos paroxítonos, de acuerdo con las unidades declarativas que fueron segmentadas.

\section{Métodos y técnicas}

El presente trabajo sigue una metodología que combina métodos del modelo de análisis comunicativo para el estudio de la entonación (García Riverón, 1996a) y el modelo métrico autosegmental. Del primero, se emplea el método auditivo (basado en criterios semánticos y acústicos) para la segmentación de las unidades del discurso (entonemas enunciativos). Dada la diversidad formal de los enunciados declarativos en muestras espontáneas se segmentaron enunciados que coinciden en su totalidad con una unidad entonativa con valor semántico enunciativo, delimitada acústicamente por pausa o inflexión final descendente de la F0.

Seguidamente, se aplicaron las técnicas de control, que como afirma García Riverón (1996a) «consisten en aislar las curvas segmentadas». Además, se acompañan de la aplicación de un nuevo análisis auditivo (directo) para comprobar la semejanza acústica entre 
ellas. Con ayuda del programa computarizado Wavelab fueron segmentadas y aisladas en archivos .wav independientes, de modo que se pudieron reagrupar de acuerdo con sus realidades acústicas.

Se procedió luego a la aplicación del método de análisis acústico experimental a cada unidad entonativa por medio del programa informático Praat para conseguir los parámetros acústicos de frecuencia fundamental o tono en cada segmento vocálico.

Fueron tomados manualmente los valores de frecuencia fundamental en hertzios y en semitonos, el primero con el fin de obtener el tono medio del informante para la definición de tonos de frontera, y el segundo, para el análisis de las unidades con datos ya estandarizados.

Los datos de los indicadores de FO, medidos en cada una de las sílabas del segmento, fueron tabulados con el programa Excel, a partir del cual se conformaron los gráficos. Se utilizó el gráfico de líneas y puntos para la frecuencia. El eje de las y muestra las magnitudes de FO (st) y el eje de las $x$, las sílabas del segmento.

Para la descripción del comportamiento del tono se represetaron los acentos tonales y los tonos de frontera, según el modelo métrico autosegmental (MA) (Pierrehumbert, 1980; Sosa, 1999, Beckman et al, 2002, Dorta Ed., 2013; Dorta Ed., 2018). ${ }^{43}$

En el modelo autosegmental la melodía se describe a partir de la sucesión de dos tipos de tonos: un tono alto $(\mathrm{H})$ y un tono bajo (L), alineados sobre las sílabas acentuadas. Estos pueden representarse en forma de secuencias monotonales o bitonales. ${ }^{44}$ Se utilizan, así los siguientes fonemas suprasegmentales, incluyendo sus variantes ${ }^{45}$ :

$/ \mathrm{L}^{*} /$ : cuando la tónica queda baja.

$/ \mathrm{H}^{*} /$ cuando la tónica queda alta o en pico y no se produce valle anterior.

$/ \mathrm{L}^{*}+\mathrm{H} /$ cuando la tónica queda baja o en ascenso, pero en este último caso, sin que le preceda una distancia significativa del valle.

44 Este modelo ha sido sometido a varias revisiones. La propuesta realizada por Beckman et al, (2002) ha sido revisada posteriormente por diferentes autores. En esta investigación se utilizan las propuestas de Dorta (Ed.) (2018).

${ }^{45}$ El sistema de acentos tonales ha tenido muchas transformaciones. En esta investigación se asume la propuesta de Dorta (ed.) $(2013,2018)$. 
Análisis sociolingüístico de la entonación en enunciados declarativos del español de Cuba y España: primer acercamiento al corpus preseea| 115

tónica. ${ }^{46}{ }^{/ \mathrm{L}+\mathrm{H}^{*} / \text { cuando se da un ascenso de la FO desde un valle hasta la }}$

Luego de medir el tono a través de medios instrumentales se le asignan valores fonológicos contrastivos de la manera siguiente: un tono $\mathrm{H}$ lo será si es más alto que el precedente, y L si presenta una altura tonal menor. ${ }^{47}$

Además de las secuencias tonales se representan también los tonos de frontera, que representan el movimiento tonal al final de la unidad entonativa, después de la última sílaba acentuada. Para este estudio se utilizan:

$/ \% \mathrm{H} /-/ \mathrm{H} \% /$ : cuando el inicio o final de la $\mathrm{FO}$ se sitúa significativamente por encima del tono medio del hablante (TM)

$/ \% \mathrm{M} /-/ \mathrm{M} \% /$ : cuando la FO inicial o final se encuentren en el Tono medio TM o muy próxima sin que la diferencia alcance el umbral de 1,5 st. Aquí se incluyen las variantes [\%MH]-MH\%] (F0 con trayectoria ascendente, pero sin llegar a superar el umbral respecto del TM) y [\%ML][ML\%] (FO con trayectoria descendente, pero no significativamente por debajo del TM).

$/ \% \mathrm{~L} / \mathrm{L} \% /$, cuando el inicio o final de la FO se encuentren significativamente por debajo del TM.

\section{Análisis acústico de los enunciados declarativos en el español de Cuba y España}

De forma general, la descripción de los enunciados declarativos en el habla de Cuba y España permite encontrar aspectos comunes como la existencia del tonema $\mathrm{L}+\mathrm{H}^{*} \mathrm{~L} \%$ y el tonema $\mathrm{L}^{*} \mathrm{~L} \%$ (Ver figs. 1,2,3,4,5).

Aunque en la mayoría de los enunciados analizados por Martín y Dorta (2018) las frases comienzan con un tono medio [\%M], se reconoce que en el español hablado en Cuba los hombres emplean un tono bajo $[\% \mathrm{~L}]$, identificado en las declarativas proparoxítonas que analizan.

\footnotetext{
${ }^{46} \mathrm{El}$ modelo incluye también las variantes $\left[{ }_{i} \mathrm{H}+\mathrm{L}^{*}\right]$ y $\left[\mathrm{L}^{*}+\mathrm{H}\right]$ cuando se da un ascenso anterior o posterior a la tónica, pero que no llega a los 1,5 st de diferencia.

${ }^{47}$ Se considera el umbral psicoacústico de 1, 5 st (Pamies Bertrán et al, 2002) para determinar la significación perceptiva de las variaciones tonales.
} 
Sin embargo, en este estudio las medias de las declarativas analizadas en ambas variantes del español muestran un inicio en un tono bajo (\% L) en hombres y en mujeres, aunque en algunos casos se evidenció también la presencia de frases con comienzos en tonos medios [\%M] (Ver figuras 1 y 7); también se identificó que en algunos casos el tono inicial en mujeres tanto de España como de Cuba es \%H, como se aprecia en los ejemplos de ambas variantes de lengua (Ver las figuras $4 \mathrm{y}$ 5).

En cuanto al segmento pretónico se aprecia diversidad. En ambas variantes de lengua se percibe que en este segmento la curva asciende hasta alcanzar el primer pico tonal en la sílaba tónica. Sin embargo, a diferencia de la descripción que se ofrece para el español peninsular (Dorta, 2007), otros casos reflejan en el segmento pretónico un descenso del tono que va del inicio a la primera sílaba tónica, y esto se da tanto en hombres como en mujeres, como puede apreciarse en los ejemplos (Fig. 1, 2, 6). En estos últimos se percibe, además, una posrealización del primer pico tonal, ya descrita por Sosa (1999) para el español de San Juan de Puerto Rico, Caracas y La Habana (fig. 4).

El movimiento ligeramente ascendente en el anacrusis, coincide con lo descrito por Martín y Dorta (2018) para las declarativas paroxítonas. Este movimiento ascendente del inicio se percibe en las declarativas de Cuba y también en las del español peninsular (Ver fig. 7 y 8).

Debe decirse que este ascenso es más pronunciado en el español de Cuba que en el español peninsular, pudiendo alcanzar para los primeros un aumento de alrededor de 4 st, mientras que en el español peninsular el ascenso inicial es más moderado con valores de alrededor de 1 semitono. Es importante, señalar, además, que el ascenso es algo más abrupto en los hombres (puede llegar hasta casi 5 st), sobre todo de la variante cubana, resultado que coincide con lo descrito por Martín y Dorta (2018) en la descripción del inicio de las declarativas en hombres y mujeres de Cuba.

El primer acento tonal presenta, generalmente, una realización bitonal $\mathrm{L}^{+} \mathrm{H}^{*}$ (ver fig. 3) y en otros casos, la realización es también bitonal $\mathrm{H}+\mathrm{L}^{*}$.

El cuerpo de las unidades analizadas es muy variable y sus características están relacionadas con la cantidad de sílabas que posee. En enunciados de hasta seis sílabas (enunciados cortos) el movimiento tiende 
a ser escalonadamente descendente y no se aprecian picos en el interior; esto es así tanto en hombres como en mujeres del español de Cuba y de España, aunque es más frecuente en esta última variante (Ver Fig. 5, 6). Sin embargo, en enunciados que describen final circunflejo, en el cuerpo se produce un ascenso gradual (de entre 1,5 y 3 st) hasta la última sílaba tónica donde comienza a bajar (Fig. 1, 2, 3).

En enunciados de entre 6 y 10 sílabas el cuerpo puede describir un movimiento con un ligero ascenso después de la tónica, pero que continúa luego descendiendo ligeramente; este movimiento casi monótono puede apreciarse en las Fig. 4 y 8 . Como puede observarse este movimiento monótono se produce generalmente en enunciados producidos por mujeres de ambas variantes, pues en los hombres se describen algunos descensos muy marcados en la postónica del interior del segmento (Ver Fig. 7) que vuelve a alzarse rápidamente en las siguiente sílaba para comenzar un descenso escalonado hasta el final.

En el caso de Cuba y en algunos casos de España (solo 2 ejemplos, ejemplo de la Fig.1 y 2), algunos enunciados, sobre todo paroxítonos describen un ascenso en la última sílaba nuclear, etiquetado como $\mathrm{L}+\mathrm{H}^{*}$ seguido de un descenso que puede ser muy marcado, aunque de manera general es moderado, cercano a 1,5st. Este movimiento alto-descendente o circunflejo registrado en el núcleo de los finales paroxítonos tiene relevancia perceptiva en algunos casos que sí supera los 1,5 st, pero en la mayoría de los ejemplos no es significativo. pues no supera el umbral perceptivo.

También en la muestra analizada se observaron coincidencias en ambas lenguas en las declarativas cortas oxítonas, caracterizadas por la presencia de un tono $L^{*}$ (Ejemplos de las figuras 4, 7, 8, 9). Este descenso final es más pronunciado en los segmentos oxítonos que en los paroxítonos, aunque de modo general no se produce un descenso muy marcado; este puede llegar en ocasiones hasta alrededor de los 3st, pero en la mayoría de las unidades el descenso final se da entre 1 y 2 st.

El tono de juntura terminal es típicamente $\mathrm{L} \%$ en todos los casos. Este tipo de juntura concuerda con la descripción presentada por Tomás Navarro Tomás para tales enunciados. 
118 | Adriana Pedrosa Ramírez, Madeleyne Bermúdez Sánchez e Raquel María García Riverón

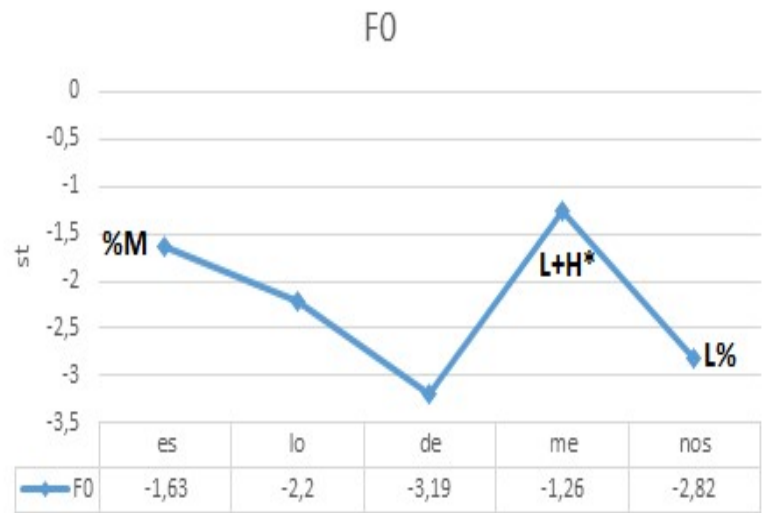

Fig. 1 Enunciado declarativo. Hombre. España

F0

0

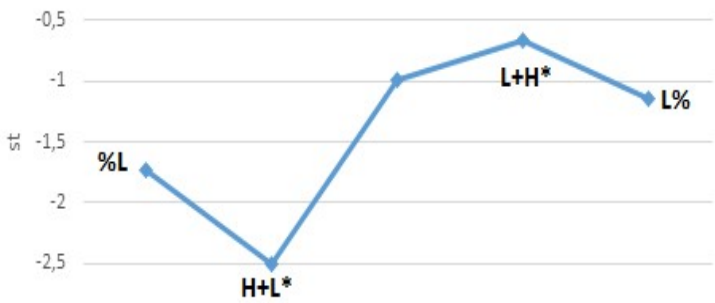

\begin{tabular}{c|c|c|c|c|c|}
\cline { 2 - 6 } & \multicolumn{1}{c|}{ ha } & bi & a & or & den \\
\hline \multirow{2}{*}{$\mathbf{F} 0$} & $-1,73$ & $-2,51$ & $-0,99$ & $-0,66$ & $-1,14$ \\
\hline
\end{tabular}

Fig. 2 Enunciado declarativo. Hombre. Cuba 
Análisis sociolingüístico de la entonación en enunciados declarativos del español de Cuba y España: primer acercamiento al corpus preseea| 119

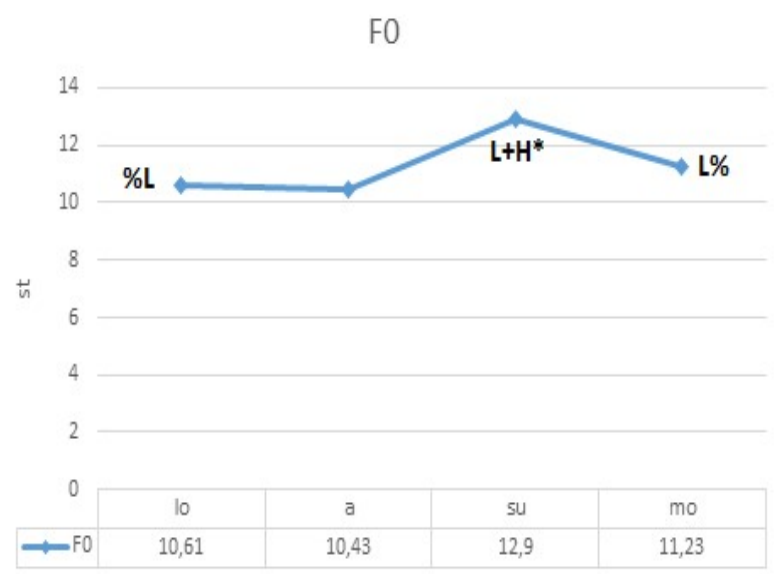

Fig. 3 Enunciado declarativo. Mujer. España

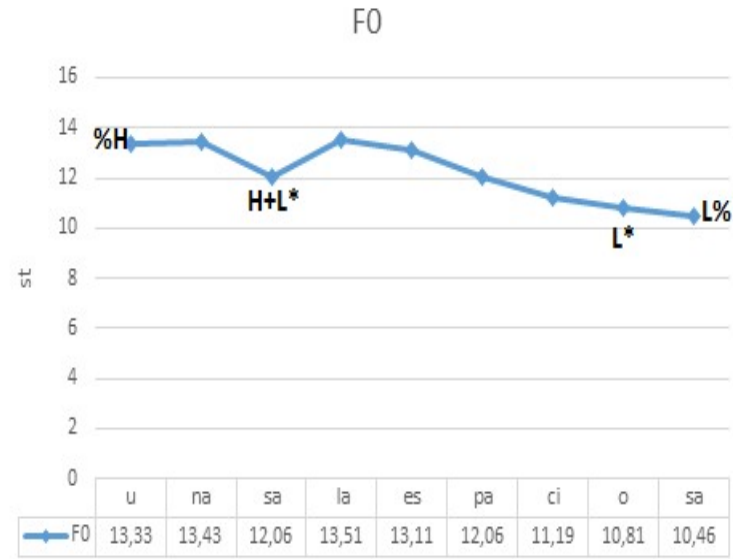

Fig. 4 Enunciado declarativo. Mujer. Cuba 
120 | Adriana Pedrosa Ramírez, Madeleyne Bermúdez Sánchez e Raquel María García Riverón

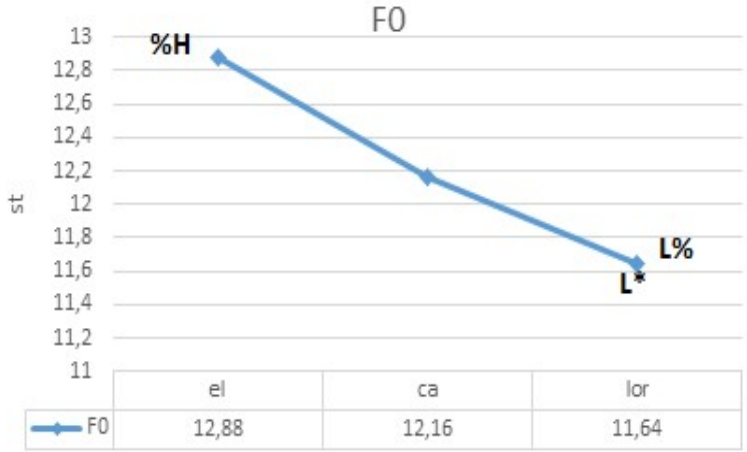

Fig. 5 Enunciado declarativo. Mujer. España

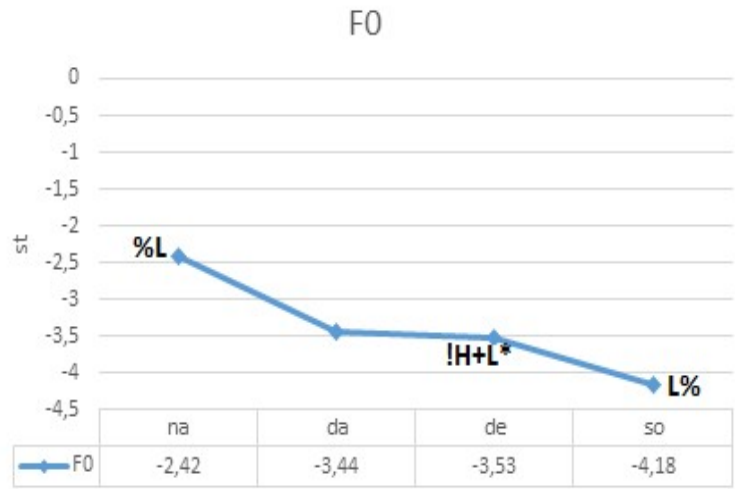

Fig. 6 Enunciado declarativo. Hombre. España 
Análisis sociolingüístico de la entonación en enunciados declarativos del español de Cuba y España: primer acercamiento al corpus preseea| 121

\section{F0}

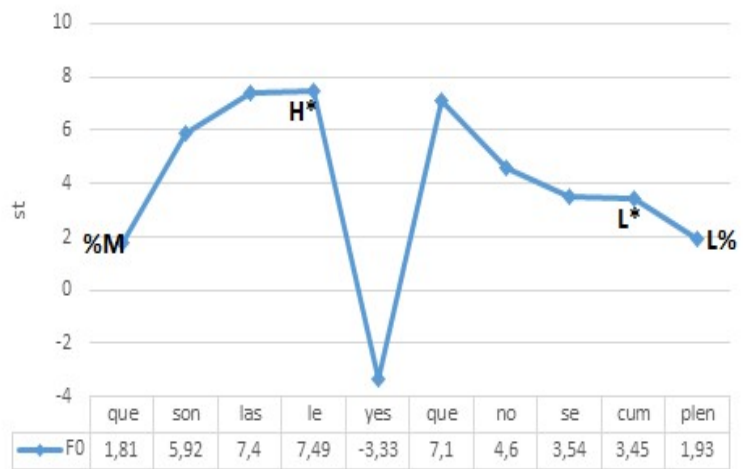

Fig. 7 Enunciado declarativo. Hombre. Cuba

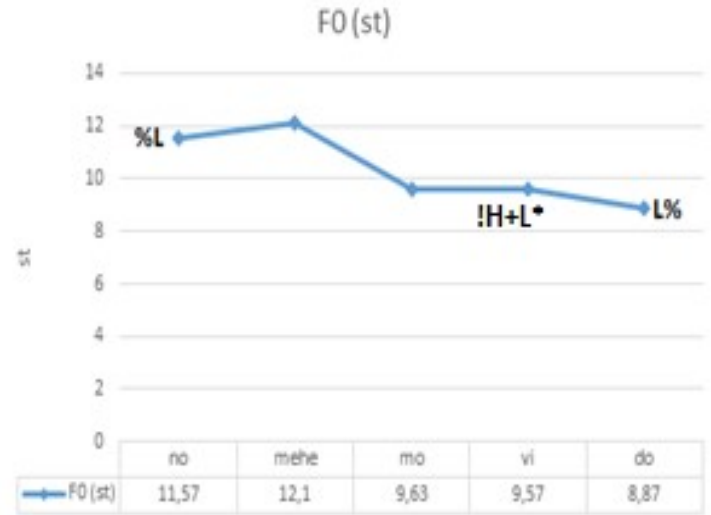

Fig.8 Enunciado declarativo. Mujer. España 
122 | Adriana Pedrosa Ramírez, Madeleyne Bermúdez Sánchez e Raquel María García Riverón

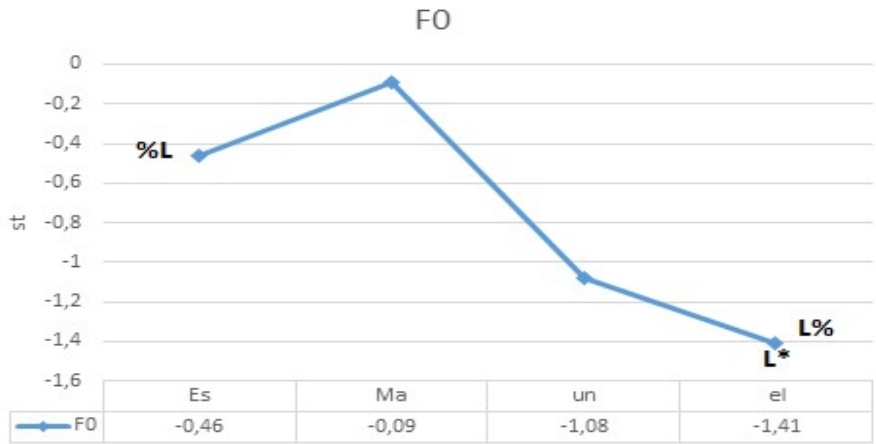

Fig. 9 Enunciado declarativo. Hombre. Cuba

\section{Consideraciones finales}

La descripción de los enunciados declarativos en el habla de Cuba y España permite encontrar aspectos comunes como la existencia del tonema $\mathrm{L}+\mathrm{H}^{*} \mathrm{~L} \%$ y el tonema $\mathrm{L}^{*} \mathrm{~L} \%$. También es común el descenso que se produce en el segmento pretonal, seguido de un movimiento bastante monótono, aunque pueden darse algunos picos o descensos en el cuerpo de las unidades, mayormente en los enunciados de los hombres de ambas variantes.

El tono de juntura terminal es $\mathrm{L} \%$ en todos los casos, coincidiendo con las descripciones expuestas desde Tomás Navarro Tomás (1968), hasta estudios más actuales como los de Martín y Dorta (2018).

\section{BIBLIOGRAFÍA}

CALLEJA APIAZU, N. (2004): Alineamiento fonético de acentos tonales en el castellano de Vitoria, Revista de Fonética Experimental VIII, 3963.

CANTERO SERENA, F. J. (2002). Teoría y análisis de la entonación. Barcelona: Ediciones de la Universidad de Barcelona.

CANTERO SERENA, F. J. \& FONT-ROTCHÉS, D. (2009). Protocolo para el análisis melódico del habla, Estudios de Fonética Experimental, XVIII, pp. 17-32. 
Análisis sociolingüístico de la entonación en enunciados declarativos del español de Cuba y España: primer acercamiento al corpus preseea| 123

CUBEIRA, M DEL C. (2003). La entonación en el habla popular de Guantánamo. Guantánamo, Cuba: Editorial El mar y la Montaña.

DORTA, J. (Ed.) (2007). Temas de Dialectología, Instituto de Estudios Canarios, La Laguna, Tenerife.

DORTA, J. (Ed.) (2013). Estudio comparativo preliminar de la entonación de Canaria, Cuba y Venezuela. Madrid- Santa Cruz de Tenerife: La página ediciones $\mathrm{S} / \mathrm{L}$, Colección Universidad. Recuperado de http: //www.researchgate.net/profile/Josefa_ Dorta/publication/271852467_estudio_comparativo_preliminar_d e_la_entonación_de_Canarias_Cuba_y_Venezuela/links 54d520aa0cf25013d02a8d55.pdf.

DORTA, J. (en prensa). La entonación interrogativa del español en la frontera México-EE.UU.

DORTA, J. (Ed.) (2018). La entonación declarativa e interrogativa en cinco zonas fronterizas del español. Canarias, Cuba, Venezuela, Colombia y San Antonio de Texas. Berna: Peter Lang.

GARCÍA RIVERÓN, R. M. (1985). La entonación de la variante cubana del español: perspectivas de la investigación. Anuario L/L (16), 256268.

GARCÍA RIVERÓN, R. M. (1989). El sistema entonativo central. Resultados preliminares. La Habana: Editorial Academia.

GARCÍA RIVERÓN, R M. (1996 a). Aspectos de la entonación hispánica. I. Metodología. Cáceres: Universidad de Extremadura.

GARCÍA RIVERÓN, M. (1996 b). Aspectos de la entonación hispánica II. Análisis acústico de las muestras del español de Cuba. Cáceres: Universidad de Extremadura.

GARCÍA RIVERÓN, R. M. (1998). Aspectos de la entonación hispánica III. Funciones de la entonación en el español de Cuba. Cáceres: Universidad de Extremadura.

GARCÍA RIVERÓN, R. M. (2002). El significado de la entonación: primer acercamiento a los datos. Oralia, (5), 3-74.

GARCÍA RIVERÓN, R. M. (2005). El estudio de la entonación. Moenia, (11), 141-176.

GARCÍA RIVERÓN, R.; BERMÚDEZ, M.; MARRERO, A. F. PEDROSA, A. (2013): Atlas Lingüístico de Cuba (ALCu), Vol. 5. Gramática y Fonética Entonación [CD- ROM]. La Habana: Academia de Ciencias de Cuba. 
HIDALGO NAVARRO, A. (2006): Aspectos de entonación española: viejos y nuevos enfoques, Cuadernos de lengua española 89, Arco/Libros,S.L.

LÓPEZ BOBO, M. J.; GONZÁLEZ RODRÍGUEZ, R.; CUEVAS ALONSO, M.; DÍAZ GÓMEZ, L.; MUÑIZ CACHÓN, C. (2005). Rasgos prosódicos del centro de Asturias: comparación OviedoMieres, en Revista de Fonética Experimental XIV, pp. 167-199.

MARTÍN BUTRAGUEÑO, P. (2015). Hacia una prosodia basada en el uso: actos de habla en el español mexicano. Revista Normas, 5, 97 115.

MARTÍNEZ CELDRÁN, E. (2005). Estudio metodológico acerca de la obtención del corpus fijo en el proyecto AMPER, en Revista de Fonética Experimental XIV, 29-66.

MARTÍNEZ CELDRÁN, E. (2007). Los dialectos catalanes y su prosodia, en Temas de Dialectología, Cap. 5, 123-141, Instituto de Estudios Canarios.

MORENO FERNÁNDEZ, F. (1998). El estudio sociolingüístico de la entonación. Oralia: Análisis del discurso oral, 1, 95-118.

MORENO FERNÁNDEZ, F. (1999). Aspectos sociolingüísticos de la entonación en el español de Alcalá de Henares (Madrid). A propósito de dos enunciados interrogativos. Publicado en A. Morales, J. Cardona, H. López Morales y E. Forastieri (eds.), Homenajea María Vaquero, Puerto Rico, Universidad de Puerto Rico, pp. 348-371.

MUÑOZ, A. (2012). Rasgos y patrones melódicos de la interrogación en el habla de Santiago de Cuba. Phonica, (8), 17-43.

MUÑOZ, A. (2013). Sistema y norma entonativos en el habla coloquial de los universitarios de Santiago de Cuba. Tesis presentada en opción al grado científico de doctor en Ciencias Lingüísticas. Cuba.

NAVARRO, T. (1968). Manual de Entonación Española. La Habana, Cuba: Instituto del Libro.

PEDROSA RAMÍREZ, A. La entonación emotiva en el español de España y Cuba. Tesis en opción al título de Licenciado en Letras. Cuba.

PIERREHUMBERT, J. \& HIRSCHBERG, J. (1990). The meaning of intonational contours in the interpretations of discourse. En Cohen, M \& Pollack (Eds.), Intention in communication (pp. 271-311), Cambridge: MIT Press. 
QUILIS, A. (1981). Fonética Acústica de la lengua española. Madrid: Gredos.

QUILIS, A. (1993). Tratado de fonología y fonética españolas. Madrid: Gredos.

RAMÍREZ VERDUGO, M.D. (2005): Aproximación a la prosodia del habla de Madrid, Revista de Fonética Experimental XIV, 309-326.

REVERT SANZ, V. (2001). Entonación y variación geográfica en el español de América, Revista Cuadernos de Filología, Anejo XLV. Universitat de València.

RODRÍGUEZ CÉSAR, D. (2005): Entonación y pragmática en la interrogación absoluta de España y Cuba, Tesis en opción al título de Licenciado en Letras. Cuba.

VELÁSQUEZ UPEGUI, E.P. (2013). Entonación del español hablado en Colombia, Tesis en opción al título de doctor en Lingüística, Centro de Estudios Lingüísticos y Literarios, El Colegio de México.

VELÁSQUEZ UPEGUI, E.P. (2016). Entonación de mandatos y ruegos en cuatro dialectos colombianos, Revista Lingüistica y Literatura No 69, 31-49, México.

Recebido em: 02/04/2019

Aceito em: 10/04/2019 